Commentary

\title{
COVID-19 associated pulmonary aspergillosis (CAPA): An added potential burden on India's pre-existing fungal superinfection
}

\section{A R T I C L E I N F O}

\section{Keywords}

Aspergillosis

COVID-19

India

Antimicrobial stewardship

Public health crises

\begin{abstract}
A B S T R A C T
The ongoing pandemic that initiated in Wuhan, China, has been an international public health emergency since January 2020. India has been battling a brutal COVID-19's second wave since April 2021. The healthcare system was struggling with a substantial increase in COVID-19 cases when the lack of necessary resources further aroused a major setback. Opportunistic fungal infections, specifically mucormycosis and candidiasis have become a pressing matter of concern. Recent cases of aspergillosis have also heightened public alarm. Hence, call for an immediate response to this public health crisis is the need of the hour by establishing countrywide surveillance, diagnostic, and management system, as well as public awareness to alleviate the burden of COVID-19 and fungal infections in India.
\end{abstract}

\section{Introduction}

COVID-19 infections and deaths have risen drastically in India. According to recent data, India is the second most affected country by COVID-19 as of 22 August 2021. The second wave in India proved to be particularly devastating with more than 400,000 daily new cases being recorded at its peak in the month of May. ${ }^{1,2}$ Since then, the COVID-19 situation has progressively worsened.

The nation recorded 325,065 active cases on 19th September 2021. However 32,740,871 people have recovered since the start of the pandemic with a current Case Fatality Ratio (CFR) of 1.34\% (down from $2.2 \%$ in June). ${ }^{1}$ The daily average of new cases was 41,383 , with hardly $11 \%$ of the population fully vaccinated in the month of September. ${ }^{3}$ Despite several steps taken to strengthen COVID-19 vaccination, ${ }^{4}$ the nation continues to be at a risk of developing superinfection such as COVID-19 associated pulmonary aspergillosis (CAPA), as seen in individuals either infected with or recovering from COVID-19.

\subsection{Burden of CAPA and other invasive fungal infections in India during the COVID-19 pandemic}

Besides COVID-19, India is suffering a mucormycosis outbreak, a lethal fungal infection that is impacting numerous COVID-19 victims. ${ }^{5-7}$ Over 45,374 mucormycosis cases and 4332 mortalities have been recorded since July of 2021. ${ }^{8}$ At least four cases of "White fungus" or candida infection have been discovered in Patna, Bihar, with many more potentially undiagnosed. ${ }^{9}$ The nation, whilst battling mucormycosis and candidiasis among COVID-19 patients, had additionally been burdened with recent outbreaks of aspergillosis in Vadodara, Gujarat. As of 27 May 2021, eight patients had been diagnosed with the ailment. ${ }^{5}$
Aspergillosis is a non-contagious fungal infection caused by Aspergillus, a common indoor and outdoor mold. The fungi are more likely to cause health concerns in immunocompromised or in individuals with chronic health debility. Symptoms include fever, cough, haemoptysis, hematemesis, chest pain and breathlessness. Additionally, it affects $20 \%-30 \%$ of mechanically ventilated COVID-19 patients. ${ }^{6,9}$ Allergies as well as invasive infections of other organs are some major health concerns produced by aspergillus. ${ }^{10}$ Most of these aspergilli are additionally resistant to azole class of drugs which could further add to the difficulty in treatment thereby hindering effective management. ${ }^{11}$

\subsection{Challenges at hand}

The number of COVID-19 patients with aspergillus co-infection is steadily increasing around the globe due to a of myriad of implicated reasons (Table 1). CAPA has been reported in over 100 patients from Europe, Asia, Australia, and South America. CAPA rates range from 4\% to $35 \%$ among the critically ill. ${ }^{12}$ The increasing incidence of triazole resistant aspergillus fungal strains in India also forms a matter of concern. ${ }^{11}$

The WHO in September 2020, advised a daily dose of $32 \mathrm{mg}$ methylprednisolone in cases of severe and critical COVID-19 whereas the Indian Directorate General of Health Services recommended 1-2 mg $/ \mathrm{kg}$ per day of the drug for severe COVID-19 infection in a person weighing $70 \mathrm{~kg}$ approximately, a dose, much higher than that recommended by the WHO. ${ }^{6}$ This has led to immunocompromised state with increased susceptibility to fungal infections. Furthermore, because India is the world's diabetes capital, the uncontrolled use of steroid-drugs among diabetics could result in an increase in CAPA cases. ${ }^{6}$ Additionally, despite mild and moderate COVID-19 manifestations, the wider

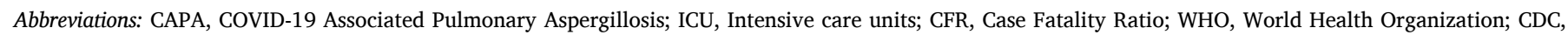

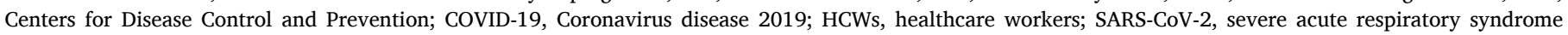
coronavirus 2 . 
Table 1

Potential causes of CAPA.

1. Damaged airway epithelium by SARS-CoV-2 ${ }^{15}$

2. Injudicious use of immune-modulating drugs such as steroids and monoclonal antibodies $^{15}$

3. Diabetic individuals, are more prone to fungal infections due to compromised leukocyte function and thereby overall immunity ${ }^{16-18}$

4. Unsanitary vents, humidifiers, vents, tubing and oxygen, which leads to direct spread through inhalation of conidia of aspergillus species resulting in a coinfection. ${ }^{5,6,9}$

populace used corticosteroids outside doctors' opinion due to anxiety. Hence, resulting in the arrival of the mucormycosis epidemic along with a severe shortage of essential drugs. The indiscriminate usage of zinc supplementation in COVID-19 sufferers is thought to be a factor in the rise in fungal infections.

Adulterant water used in oxygen therapy humidifiers, industrialized oxygen, nonsterile hospital instruments, prolonged usage with same catheter, may result not only in fungal infections but also in other nosocomial infections. The unhygienic environment slums coupled with the dilapidated state of the rural hospitals are few factors aggravating the fungal outbreak. Also, the hot and humid climate of South Asia is favorable for the growth of these fungi.

Another plaque lay in the necessity of interdisciplinary management guidelines owing to the fact that CAPA is not limited to a single speciality. ${ }^{7}$ To add to these challenges, India's already fragile and strained healthcare system is overwhelmed with the burden of several other infectious diseases. ${ }^{13,14}$ Accompanying these are frequent exposure to workplace violence inflicted upon the healthcare workers (HCW) that only offers burnout leaving psychological impacts that often bereaves the patient of treatment. Hence, an overlapping crisis of CAPA and COVID-19 can exacerbate existing burdens and may even result in collapse of the healthcare system as CAPA is additionally shown to affect both immunosuppressed as well as immune-competent individuals. ${ }^{11}$

\subsection{Recommendations}

Given the gravity of an unruly situation, immediate efforts by policy makers and relevant stakeholders to contain CAPA is required at the earliest to prevent another outbreak like that of mucormycosis.

Guidelines which have been laid down by the likes of healthcare authorities such as the $\mathrm{CDC}$ and the WHO about the proper regulation and monitoring of drug dosages and chronic ailments should be followed. To prevent the development of Aspergillosis, the authorities must resolve the country's lack of medications and teach a large number of people to build technical skills. ${ }^{19}$ As a solution to ensure requisite supply chain of medicines, relaxation on imports may be implemented.

Government mandated health promotion and awareness campaigns as community outreach programs with the help of educational institutions and organizations may go a long way in helping combat the emerging crisis. Mass and outreach vaccination should be made a priority, particularly in the containment zones. The community field guide workers can make the people aware about health and hygiene, maintenance of clean surroundings, proper handwashing, mask usage, besides addressing their concerns for vaccination. Continuous periodic assessments to ensure quality check of oxygen supplied to hospitals, besides, inspection of other drugs and overall hospital environment should be introduced. A close monitoring and epidemiological surveillance using contemporary technology, should be emphasized upon. The authorities should also promote research on different diseases and provide financial grants for the development of technologies that can aid in their prevention as well as treatment.

\section{Conclusion}

Thus, with a surge in the incidence rate of fungal and COVID-19 infections across India, it is vital to take precautionary measures and adhere to the recommended guidelines. Collegial efforts of continuous diagnosis, management, and surveillance, besides, public understandings are the way to manage the overlapping crisis of CAPA in India.

\section{Author contributions}

All authors substantially contributed to the preparation of this article. All authors revised and approved the final draft.

\section{Funding}

No external funding was used in this study.

\section{Availability of data and materials}

Not applicable.

\section{Ethics approval and consent to participate}

Not applicable.

\section{Declaration of competing interest}

The authors declare that there is no conflict of interests.

\section{Acknowledgments}

Not applicable.

\section{References}

1. India: coronavirus pandemic country profile - our world in data (n.d.) https://ourwo rldindata.org/coronavirus/country/india. Accessed September 20, 2021.

2. Ghosh S, Moledina N, Hasan MM, Jain S, Ghosh A. Colossal challenges to healthcare workers combating the second wave of coronavirus disease 2019 (COVID-19) in India. Infect Control Hosp Epidemiol. 2021:1-2. https://doi.org/10.1017/ ICE.2021.257.

3. The New York Times. India coronavirus map and case count. https://www.nytimes. com/interactive/2021/world/india-covid-cases.html; 2021. Accessed September 20, 2021.

4. Rackimuthu S, Hasan MM, Bardhan M, Essar MY. COVID-19 vaccination strategies and policies in India: the need for further re-evaluation is a pressing priority. Int $J$ Health Plann Manag. 2021. https://doi.org/10.1002/HPM.3321.

5. Ghazi BK, Rackimuthu S, Wara UU, et al. Rampant increase in cases of mucormycosis in India and Pakistan: a serious cause for concern during the ongoing COVID19 pandemic. Am J Trop Med Hyg. 2021;1. https://doi.org/10.4269/AJTMH.210608.

6. Rocha ICN, Hasan MM, Goyal S, et al. COVID-19 and mucormycosis syndemic: double health threat to a collapsing healthcare system in India. Trop Med Int Health. 2021;26:1016-1018. https://doi.org/10.1111/TMI.13641.

7. Rackimuthu S, Khan H, Mohan A, et al. Emergence of a medley of invasive fungal infections amidst the coronavirus disease 2019 (COVID-19) pandemic in India. Antimicrob. Steward. Healthc. Epidemiol. 2021;1:e40. https://doi.org/10.1017/ ASH.2021.198.

8. India News. Times of IndiaIndia reports 45,374 Black fungus cases, 4,332 deaths so far, says health ministry. https://timesofindia.indiatimes.com/india/india-reports45374-black-fungus-cases-4332-deaths-so-far-says-health-ministry/articleshow/846 40357.cms; 2021. Accessed September 20, 2021.

9. Ghosh S, Patelia S, Hasan MM, Ghosh A, Jain S, Patel T. Drug resistant White fungus: another catastrophic fungus emergence amidst COVID-19 in India, 10.1080/ 20477724.2021.1960762 https://doi.org/10.1080/20477724.2021.1960762; 2021.

10. CDC. Types of fungal diseases (n.d.) https://www.cdc.gov/fungal/diseases/index. html. Accessed September 20, 2021.

11. Meijer EFJ, Dofferhoff ASM, Hoiting O, Buil JB, Meis JF. Azole-resistant COVID-19associated pulmonary aspergillosis in an immunocompetent host: a case report, 2020 J. Fungi. 2020;6:79. https://doi.org/10.3390/JOF6020079, 79. 6.

12. Hoenigl M. Invasive fungal disease complicating coronavirus disease 2019: when it rains, it spores. Clin Infect Dis. 2020. https://doi.org/10.1093/CID/CIAA1342.

13. Bardhan M, Pramanik D, Riyaz R, Hasan MM, Essar MY. Dual burden of Zika and COVID-19 in India: challenges, opportunities and recommendations. Trop Med Health. 2021;49:1-4. https://doi.org/10.1186/S41182-021-00378-0/METRICS.

14. Bardhan M, Hasan MM, Ray I, et al. Tuberculosis amidst COVID-19 pandemic in India: unspoken challenges and the way forward. Trop Med Health. 2021;49:1-5. https://doi.org/10.1186/S41182-021-00377-1/METRICS. 
15. A W, I S. Editorial MMCR special issue "Covid-19 associated pulmonary aspergillosis. Med. Mycol. Case Rep. 2021;31:1. https://doi.org/10.1016/J. MMCR.2021.02.003.

16. Rodrigues CF, Rodrigues ME, Henriques M. Candida sp. infections in patients with diabetes mellitus. J Clin Med. 2019;8:76. https://doi.org/10.3390/JCM8010076.

17. Essar MY, Khan H, Babar MS, et al. Mucormycosis, conflicts and COVID-19: a deadly recipe for the fragile health system of Afghanistan. Int $J$ Health Plann Manag. 2021. https://doi.org/10.1002/HPM.3292.

18. Asri S, Akram MR, Hasan MM, et al. The risk of cutaneous mucormycosis associated with COVID-19: a perspective from Pakistan. Int J Health Plann Manag. 2021:3311. https://doi.org/10.1002/HPM.3311. hpm.

19. Arun AB, Hasan MM, Rackimuthu S, Ullah I, Mir T, Saha A. Antifungal drug shortage in India amid an increase in invasive fungal functions during the coronavirus disease 2019 (COVID-19) pandemic. Infect Control Hosp Epidemiol. 2021:1-2. https://doi. org/10.1017/ICE.2021.426.

Priyanka Mohan Lal Ziauddin Medical University, Karachi, Pakistan

Aabiya Arif

Ziauddin Medical University, Karachi, Pakistan E-mail address: aabiya.arif@gmail.com.

Anmol Mohan

Karachi Medical \& Dental College, Karachi, Pakistan

E-mail address: anmolmohanvan@gmail.com.

Sudhan Rackimuthu

Father Muller Medical College, Mangalore, Karnataka, India

E-mail address: sudhan.racki@gmail.com.
Mohammad Mehedi Hasan

Department of Biochemistry and Molecular Biology, Faculty of Life Science, Mawlana Bhashani Science and Technology University, Tangail, Bangladesh Division of Infectious Diseases, The Red-Green Research Centre, BICCB,

Dhaka, Bangladesh

E-mail address: mehedi.bmb.mbstu@gmail.com.

Zarmina Islam

Dow University of Health Sciences, Karachi, Pakistan E-mail address: zarmina2001@gmail.com.

Utkarsha Uday

West Bengal University of Health Sciences, Kolkata, West Bengal, India E-mail address: utkarsha.tanuday@gmail.com.

Um-Ul- Wara

Karachi Medical \& Dental College, Karachi, Pakistan

E-mail address: umulwara28@gmail.com.

Muhammad Taha Arshad Shaikh

Karachi Medical \& Dental College, Karachi, Pakistan E-mail address: tahashaikh96@gmail.com.

Mohammad Yasir Essar Kabul University of Medical Sciences, Kabul, Afghanistan

* Corresponding author. E-mail address: yasir.essar@gmail.com (M.Y. Essar). 